\begin{tabular}{|r|r|r|r|r|}
\hline$s$ & $|f(s)|^{\mathrm{a}}$ & $|f(s)|^{\mathrm{b}}$ & $\eta(s)^{\mathrm{a}}$ & $\eta(s)^{\mathrm{b}}$ \\
\hline 0 & 12,3468 & 12,3564 & 0,335 & 0,334 \\
5 & 3,4072 & 3,4100 & 0,953 & 0,950 \\
10 & 1,3555 & 1,3521 & 1,788 & 1,782 \\
15 & 0,7774 & 0,7740 & 2,443 & 2,438 \\
20 & 0,5080 & 0,5055 & 2,985 & 2,979 \\
25 & 0,3562 & 0,3540 & 3,454 & 3,449 \\
30 & 0,2605 & 0,2587 & 3,868 & 3,867 \\
35 & 0,1960 & 0,1949 & 4,255 & 4,258 \\
\hline
\end{tabular}

Tab. 5. Gegenüberstellung der bei zwei verschiedenen Integrationsschrittweiten erhaltenen Werte der Streuamplituden von Uran $(40 \mathrm{kV})$, a) Schrittweite $h=2 \cdot 10^{-4}$, b) Schrittweite $h=2 \cdot 10^{-3}$.

Die Berechnung der Funktionen $K_{0}(u)$ und $K_{1}(v)$ wurde nach Näherungsformeln durchgeführt, die dem "Handbook of Mathematical Functions" ${ }^{14}$ entnommen wurden.
Zur Berechnung der Legendre-Polynome wurde die bekannte Rekursionsformel verwendet. Um die Konvergenz der Reihe für $f(\vartheta)$, Gl. (1), zu verbessern, wurden die von Hoerni und Ibers ${ }^{3}$ angegebenen Konvergenzkorrekturen angewendet.

In der Weiterführung dieser Arbeit sollen zunächst für alle Elemente des periodischen Systems die Beträge und Phasen der Streufaktoren berechnet und dann durch analytische Ausdrücke angenähert werden, um sie bei ihrer weiteren Verwendung auf dem Gebiet der Elektronenbeugung an Gasen in möglichst einfacher Form vorliegen zu haben.

Ich danke Herrn Prof. Dr. W. ZeIL für anregende Diskussionen und sein stets förderndes Interesse an dieser Arbeit.

14 Handbook of Mathematical Functions, NBS Applied Math. Series 55.

\title{
Die Ionenladungsverteilung von unabgebremsten Spaltprodukten
}

\author{
E. Konecny und G. Siegert
}

\author{
2. Physikalisches Institut der Justus-Liebig-Universität Gießen \\ und Physik-Department der Technischen Hochschule München
}

(Z. Naturforschg. 21 a, 192-196 [1966]; eingegangen am 8. November 1965)

\begin{abstract}
Eine frühere Arbeit über die Ionenladungsverteilung von Spaltprodukten ${ }^{1}$ wurde in zwei Punkten ergänzt. Einmal wurden die Spaltprodukte aller natürlich vorkommenden Energien und Ionenladungen erfaßt und mit den integralen früheren Messungen von LASSEN ${ }^{2}$ verglichen. Zum anderen wurden unter Benützung des gesamten Massenspektrographen am FRM in Garching bei München Ionenladungsverteilungen bei konstanter kinetischer Energie und Masse aufgenommen und die Abhängigkeit der mittleren Ionenladung als Funktion der Geschwindigkeit bei konstanter Masse untersucht.
\end{abstract}

In einer früheren Arbeit ${ }^{1}$ wurde die Ionenladung von Spaltprodukten in Abhängigkeit von deren kinetischer Energie untersucht. Zur Analyse diente ein elektrostatisches Ablenkfeld, das die Teilchen nach den Quotienten von kinetischer Energie $E$ und Ionenladungszahl $e$ sortiert. Aus technischen Gründen konnte damals die Ablenkfeldstärke nicht genügend hoch gemacht werden, um den ganzen bei der Spaltung natürlicherweise auftretenden Energiebereich zu erfassen. Teilchen mit hohen Energien und tiefen Ionenladungen der leichten Spaltproduktgruppe konnten nicht mehr abgelenkt werden. Die hier aufgeführten Messungen sollen die Arbeit ${ }^{1}$ in zweifacher Hinsicht ergänzen:

1 H. Opower, E. Konecny u. G. Siegert, Z. Naturforschg. 20 a, $131[1965]$. a) Auch die hochenergetischen Teilchen der leichten Gruppe werden einbezogen; unter Verwendung dieser Daten ist ein direkter Vergleich unserer Ergebnisse mit früheren Daten von LASSEN ${ }^{2}$ für die jeweils über die leichte und schwere Gruppe integrierte Ionenladungsverteilung möglich.

b) Bei der Trennung nur durch ein elektrostatisches Feld kann man nur nach der Energie, nicht aber zusätzlich nach der Masse trennen. Unter zusätzlicher Benützung eines homogenen magnetischen Ablenkfeldes wurden hier die Häufigkeitsverteilungen der Ionenladungen von Spaltprodukten einzelner diskreter Massen bei festen kinetischen Energien untersucht.

2 N. O. Lassen, Kgl. Danske Vidensk. Selskab, Mat. Fys. Medd. 26, Nr. 5 [1951]. 
Die hier weitergegebenen Meßdaten wurden erhalten als Nebenprodukte zweier anderer Arbeiten, und zwar (Teil a) von Untersuchungen über den Ionisationsdefekt von Festkörpersperrschichten ${ }^{3}$ und (Teil b) von Untersuchungen über Feinstrukturen in den Häufigkeitsverteilungen von Masse und Energie bei der thermischen Spaltung von $\mathrm{U}^{235}{ }^{4}$. Als Versuchsanordnung diente hierzu der am Reaktor FRM in München aufgebaute Massenspektrograph für Spaltproduktstrahlen, der in $\mathbf{5}$ ausführlich beschrieben ist. Eine schematische Skizze hiervon zeigt Abb. 1. Als Strahlquelle diente eine $100 \mu \mathrm{g} / \mathrm{cm}^{2}$ dicke $\mathrm{U}^{235}$.Folie im Neutronenfluß des Re-

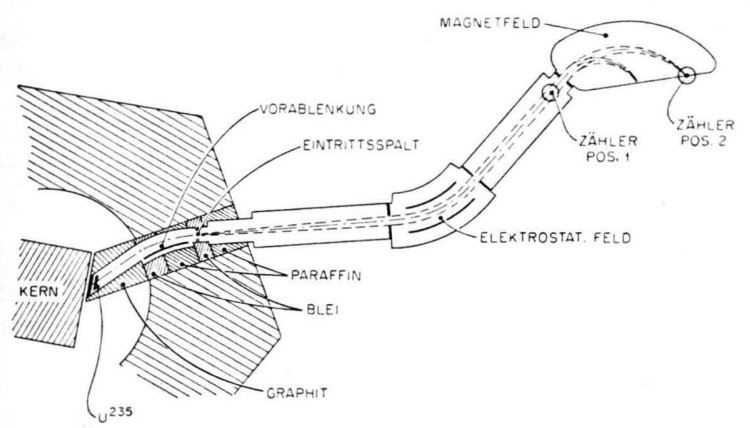

Abb. 1. Schematische Skizze des Versuchsaufbaues am FRM.

aktors. Die sogen. Vorablenkung dient nur zum Herauslenken des Spaltproduktstrahls aus störendem $\gamma$ - und Neutronenuntergrund. Erst dahinter beginnt mit dem Eintrittsspalt der eigentliche Spektrograph. Die Registrierung der Teilchen erfolgte für den Teil a) mittels eines Festkörpersperrschichtzähhlers am Ort 1 in Abb. 1 unmittelbar vor dem Eintritt des Strahls ins Magnetfeld oder für den Teil b) am Ort 2 mittels Kernspurplatten (Typ Ilford KO) oder mit Festkörperzählern. Eine reproduzierbare und im Relativwert genaue Einstellung der Feldstärke im Toroidkondensator geschah über eine Kompensationsmessung der Spannung unter Vorschaltung eines in Öl befindlichen Hochspannungsteilers ${ }^{1}$, eine genaue Messung des Magnetfeldes mit einem Kernresonanz-Magnetfeldmesser.

Die Messungen für den Teil a) wurden vollkommen analog wie in der Arbeit ${ }^{1}$ durchgeführt, nur daß hier zur Erreichung höherer elektrischer Feldstärken bei gleicher Ablenkspannung der Elektrodenabstand enger (auf 4,5 cm) gestellt wurde. Aus dem Gleichsetzen von elektrischer Ablenkkraft und Zentrifugalkraft ergibt sich für die Sollbahn der Teilchen die einfache Beziehung

$$
E / e=b \cdot F
$$

mit $E=$ kinetischer Energie der Teilchen, $e=$ Ionenladungszahl ( = Ionenladung in Einh. der Elementar-

3 E. Konecny u. K. Hetwer, Nucl. Instr. Methods 36, 61 [1965].

4 In Vorbereitung. ladung $\left.e_{0}\right), F=$ Ablenkfeldstärke. $b$ ist eine apparative Konstante, deren exakter Wert durch Eichung mit $\mathrm{Po}^{210}-\alpha$-Teilchen, deren Energie und Ionenladungszahl bekannt sind, ermittelt wurde. Zusammen mit einer unabhängigen Bestimmung der Ionenladungszahl, wie sie in ${ }^{1}$ ausführlich beschrieben ist, ist damit eine absolute, auf etwa $3 \cdot 10^{-3}$ genaue Bestimmung der Energie möglich. Die Halbwertsbreite der vom Kondensator durchgelassenen Energie beträgt etwa $1 \%$. Bei 20 verschiedenen Spannungseinstellungen, die so gewählt wurden, daß der gesamte Bereich, in dem Spaltprodukte vorkommen, überstrichen wurde, ergaben sich Spektren verschiedener diskreter Linien, die zu verschiedenen Ionenladungen gehören, analog zu Abb. 2 in ${ }^{1}$. Abb. 2 in der jetzigen Arbeit zeigt die mittleren Energien der

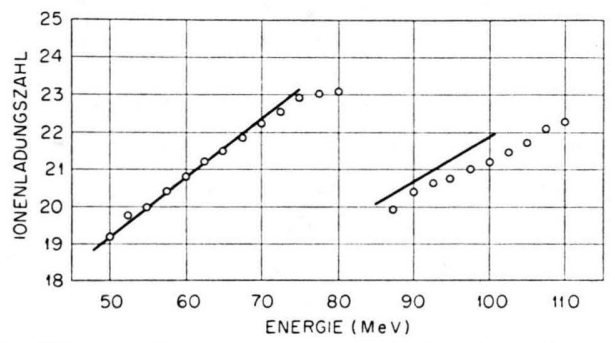

Abb. 2. Die am Ort 1 gemessene mittlere Ionenladung der Spaltprodukte als Funktion ihrer Energie (০). Die Linien geben die Meßwerte der Arbeit ${ }^{1}$ an.

Ionenladungsverteilungen zusammen mit den Werten aus Arbeit ${ }^{1}$. Für die schwere Gruppe zeigt sich vollkommene Übereinstimmung mit den in der Arbeit ${ }^{1}$ wiedergegebenen Daten. Wegen der unvollkommenen Einbeziehung der niedrigen Ionenladungszahlen ist die mittlere Ladung in der leichten Gruppe in unserer ersten Untersuchung etwa um ca. 0,4 Ladungseinheiten zu hoch geraten. Diese werden ja nach Gl. (1) nur bei den höchsten Feldstärken erfaßt. Zum Vergleich der Daten mit früheren, von Lassen ${ }^{2}$ mittels Ablenkung der Spaltfragmente in einem Magnetfeld bestimmten Daten, wurde über die Energie jeweils der leichten und schweren Gruppe der Spaltprodukte integriert. Das Ergebnis ist zusammen mit dem aus den Lassenschen Daten gewonnenen Ergebnis (aus einer Kombination der Abb. 4 und 12 in ${ }^{2}$ ) in Abb. 3 aufgetragen. Unsere Kurven erweisen sich dabei als geringfügig schmaler. Die mittlere Ladung der leichten Teilchen liegt um eine Einheit der Elementarladung $e_{0}$ höher als bei LASSEN angegeben.

5 H. Ewald, E. Konecny, H. Opower u. H. Rösler, Z. Naturforschg. 19 a, 194 [1964]. 


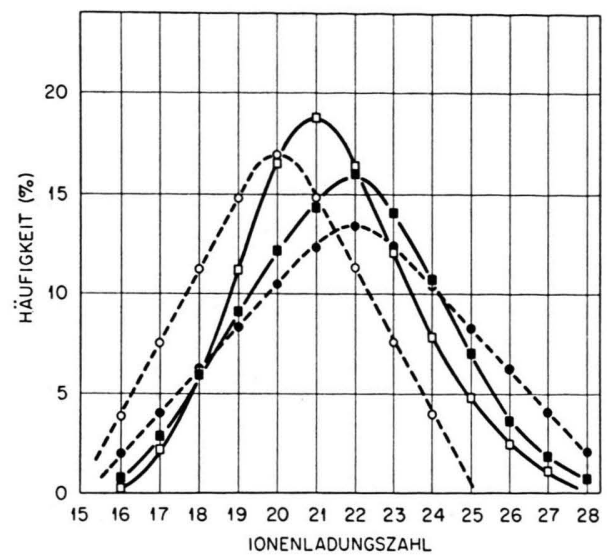

Abb. 3. Die Ionenladungsverteilung der Spaltprodukte, jeweils über alle Teilchen der leichten $(\square)$ und der schweren (匹) Gruppe integriert (ausgezogene Linien). Gestrichelt sind zum Vergleich die entsprechenden Werte von Lassen ${ }^{2}$ angegeben ( $\circ$ leichte Gruppe, - schwere Gruppe).

Für den Teil b) wurde der ganze Massenspektrograph verwendet. Zur Ablenkgleichung (1) kommt noch eine ähnliche durch Gleichsetzen von Zentrifugalkraft für die betrachtete Bahn im Magnetfeld und LoRENTz-Kraft hinzu; durch Elimination der Teilchengeschwindigkeit aus diesen beiden Gleichungen folgt dann

$$
M / e=\text { const. } \varrho^{2} \cdot B^{2} / F
$$

mit $M=$ Massenzahl (= Masse in Einheiten der atomaren Masseneinheit), $B=$ magnetische Induktion. $\varrho$ ist der Abstand des Bildortes der betrachteten Massenlinie vom Eintrittspunkt des Strahls in das Magnetfeld. Aus den ionenoptischen Eigenschaften des Massenspektrometers (Mattauch-Herzog-Apparat) ${ }^{5}$ folgt nämlich, daß die Bildebene den Eintrittspunkt einschließt.

Man erhält also nach Gl. (2) durch die Vielfältigkeit der Ionenladungszahlen $e$ im Prinzip verschiedene überlagerte Massenspektren. Infolge einer bestehenden Beziehung zwischen Energie und Masse bei den primären Spaltprodukten ist aber in der schweren Gruppe eine Trennung nach Massen allein möglich, wie an anderer Stelle ${ }^{6}$ ausführlich beschrieben ist. Zur Bestimmung der Verteilungskurven der Ionenladung bei verschiedenen Massen und Energien wurden in der Bildebene Kernspurplatten mit einer Länge von etwa $23 \mathrm{~cm}$ für je $12 \mathrm{~h}$ exponiert. Nach Auszählen der Spaltproduktspuren in je $160 \mu$ breiten Streifen von $10 \mathrm{~mm}$ Länge mit je $500 \mu \mathrm{Ab}$ -

6 E. Konecny, H. Opower u. H. Ewald, Z. Naturforschg. 19a, 200 [1964]. stand, die parallel zu den Massenlinien verlaufen, ergeben sich $M / e$-Spektren, wie Abb. 4 als Beispiel eines zeigt. Man sieht dort periodisch wiederkehrende Liniengruppen, die einzelnen Ionenladungen und damit auch verschiedenen Energien angehören. Ionenladungszahl (in Einheiten der Elementarladung $e_{0}$ ) und kinetische Energie sind jeweils über die Liniengruppen geschrieben, die Masse, die der Linie zugeordnet wurde, steht darunter. Da der gezeigte Ausschnitt nur eine Länge von etwa $23 \mathrm{~cm}$ der Bildebene bei einem mittleren Abstand von ca. $150 \mathrm{~cm}$ vom Eintrittspunkt umfaßt, ist von dem quadratischen Verlauf von $M$ mit $\varrho$, wie er nach Gl. (2) auftreten muß, fast nichts zu sehen.

Insgesamt wurden fünf solcher Aufnahmen ausgewertet. Das elektrische Feld wurde hierbei so eingestellt, daß jeweils Teilchen einer bestimmten festen Energie $E_{1}$ bei den aufeinanderfolgenden Einstellungen den Kondensator mit den Ionenladungszahlen $e_{1}+i(i=1,2,3, \ldots)$ passieren konnten, d. h.

$$
F_{i}=F_{1} e_{1} /\left(e_{1}+i-1\right) .
$$

Für diesen Satz von Messungen wurde hierbei $E_{1}=77,7 \mathrm{MeV}$ (charakteristisch für den Massenbereich um $M=134$ ) und $e_{1}=22$ gewählt. Das Magnetfeld wurde für jede Messung so eingestellt, $\mathrm{da} ß$ das gleiche $M / e$ für alle Messungen am gleichen Ort erschien, d. h. $B^{2} / F$ wurde konstant gehalten.

Um quantitativ festzustellen, wie groß bei den einzelnen Spektrallinien der Abb. 4 die Beimengungen von anderen Massen von benachbarten Ionenladungen mit gleichem $M / e$ ist, wurden mit einem Festkörpersperrschichtzähler am Ort der Spektrallinien mittels eines Vielkanalanalysators die Energiespektren aufgenommen: Verschiedene Ionenladungen haben gemäß Gl. (1) auch verschiedene, um etwa 5\% unterschiedliche, diskrete Energien, die bequem zu trennen sind ${ }^{3}$. Aus Gründen experimenteller Einfachheit wurden hierbei die jeweils untersuchten Linien bei konstantem Ort durch nach Gl. (2) entsprechende Änderung des Magnetfeldes eingestellt.

Die Zuordnung der Ionenladungen zu den Spektrallinien in Abb. 4 geschah einmal durch Intensitätsvergleich mit den am Ort 1 (Abb. 1) aufgenommenen Ionenladungsspektren, für die die Ionenladungen nach der beschriebenen Methode bestimmt worden waren, und davon unabhängig durch Ausmessen der Linienabstände der Spektrallinien derselben Masse benachbarter Ionenladung, wie in ${ }^{6}$ ausführlich beschrieben. Die Massenzuordnung wurde durch 


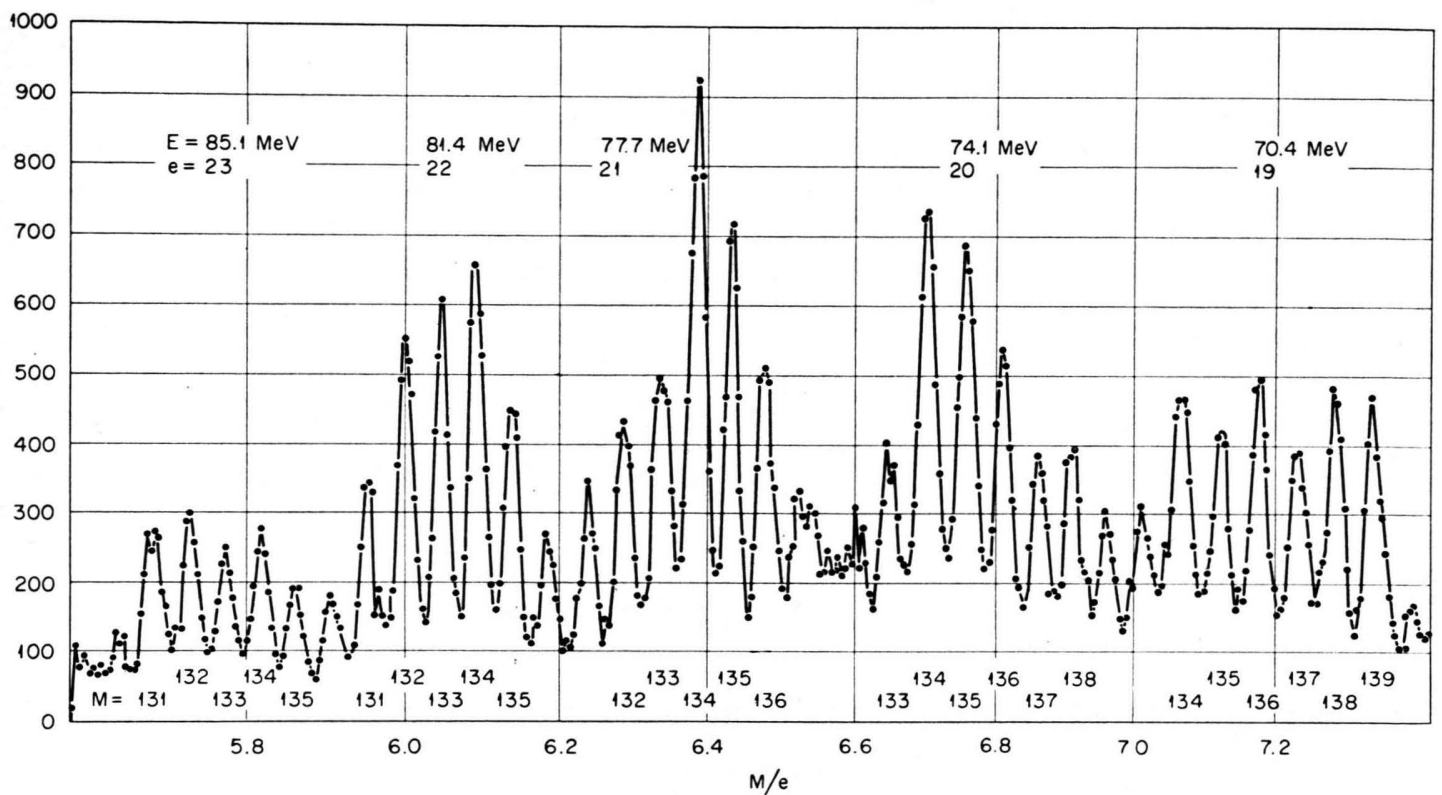

Abb. 4. In der Bildebene des Spektrographen erhaltenes $M / e$-Spektrum für eine Einstellung des elektrischen Feldes entsprechend $E / e=3,705 \mathrm{MeV} /$ Ladungseinheit. Über die einzelnen Liniengruppen sind Energie und Ionenladungszahl (in Einheiten der Elementarladung) geschrieben, unter die einzelnen Linien die zugehörige Masse. Nähere Erklärung im Text. Das Spektrum wurde erhalten durch Auszählen der Spaltproduktspuren in einer Kernspuremulsion, die in der Bildebene des Spektrographen exponiert worden war. Der Abstand zweier benachbarter Linien beträgt etwa $5,5 \mathrm{~mm}$.

Eichung des Apparates mit $\mathrm{Po}^{210}$ a-Teilchen und durch Intensitätsvergleich ${ }^{6}$ der erhaltenen Massenspektren mit radiochemischen Ausbeutedaten durchgeführt.

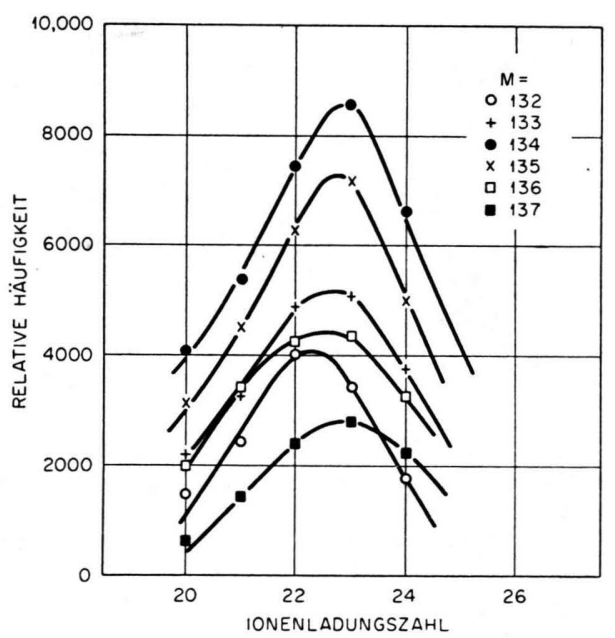

Abb. 5. Ionenladungsverteilung einzelner Spaltproduktmassen der schweren Gruppe $(M=132$ bis 137$)$ bei konstanter kinetischer Energie (77,7 MeV). Die Geschwindigkeit der Spaltprodukte ist dabei ebenfalls nahezu konstant, sie ändert sich von $1,06 \cdot 10^{9} \mathrm{~cm} / \mathrm{sec}$ für $M=132$ bis $1,04 \cdot 10^{9} \mathrm{~cm} / \mathrm{sec}$ für $M=137$.
Abb. 5 zeigt die Ionenladungsverteilung der Spaltprodukte mit den Massenzahlen 132 bis 137 bei der kinetischen Energie 77,7 MeV. Das Ergebnis sind GAUss-ähnliche Kurven, die allerdings nach höheren Ionenladungen etwas steiler abfallen. Die Halbwertsbreite beträgt 4,5 $\pm 0,3$ Ladungseinheiten.

Abb. 6 gibt für den kleinen, mit den 5 Aufnahmen erfaßten Energiebereich die häufigste Ionenladung für Spaltprodukte einer Massenzahl $(M=134)$ in Abhängigkeit von der Geschwindigkeit (oberer Maßstab) bzw. von der kinetischen Energie (unterer Maßstab) an.

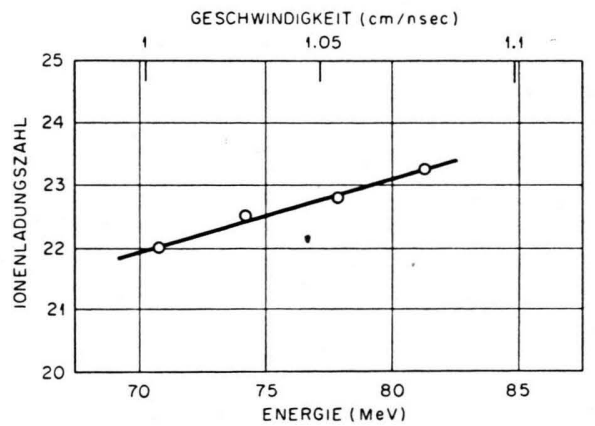

Abb. 6. Abhängigkeit der mittleren Ionenladung von der Energie (untere Skala) bzw. Geschwindigkeit bei konstanter Masse $M=134$. 
Die Verteilungskurven bei konstanter Masse und Energie erweisen sich als durchweg schmaler als in ${ }^{1}$ abgeschätzt; der Grund hierfür liegt wahrscheinlich an dem dort verwendeten relativ dicken (und inhomogenen) Uranpräparat. Ein Hinweis hierfür ist auch die über alle Energien und Massen gemittelte schmalere Ionenladungsverteilung in diesem Experi-

\begin{tabular}{|c|c|c|c|}
\hline Gruppe & $\mathrm{L}^{2}$ & OKS 1 & $\begin{array}{c}\text { dieses } \\
\text { Experiment }\end{array}$ \\
\hline $\begin{array}{l}\text { leichte Gruppe } \\
\text { schwere Gruppe }\end{array}$ & $\begin{array}{l}5,5 \mathrm{MeV} \\
7,4 \mathrm{MeV}\end{array}$ & $\begin{array}{l}4,8 \mathrm{MeV} \\
7,3 \mathrm{MeV}\end{array}$ & $\begin{array}{l}4,7 \mathrm{MeV} \\
6,0 \mathrm{MeV}\end{array}$ \\
\hline
\end{tabular}

Tab. 1. Halbwertsbreiten der über alle Energien und Massen jeweils einer Spaltproduktgruppe gemittelten Ionenladungsverteilung. ment. Zum Vergleich gibt Tab. 1 die Halbwertsbreiten über alle Teilchen jeder Gruppe von dieser Arbeit mit den in ${ }^{1}$ abgeschätzten (OKS) und denen von LASSEN ${ }^{2}(\mathrm{~L})$ an. Von diesen Änderungen bleibt aber die in ${ }^{1}$ gegebene generelle Diskussion der Abhängigkeit der mittleren Ionenladung von Geschwindigkeit und Masse unberührt.

Herrn Prof. H. Ewald danken wir für die stete Förderung, Herrn Prof. H. Maier-Leibnitz für sein Interesse an der Arbeit. Den Kollegen unserer Arbeitsgruppe, Dipl.-Phys. H. Gunther, Dipl.-Phys. H. RösLer, cand. phys. R. Gebert, H. Göbel, K. Hetwer, K. Kürzinger und R. Winter, schulden wir Dank für die Mithilfe bei den Messungen, Frl. H. Rehse für den Großteil der mikroskopischen Auswertung.

\title{
Massenspektrometrische Untersuchung der Photoionisation von Molekülen
}

\author{
Burkhard Brehm* \\ Physikalisches Institut der Universität Freiburg i. Br.
}

(Z. Naturforschg. 21 a, 196-209 [1966] ; eingegangen am 4. Oktober 1965)

\begin{abstract}
Für die Atome $\mathrm{Xe}$ und $\mathrm{Hg}$ und die Moleküle $\mathrm{O}_{2}, \mathrm{H}_{2} \mathrm{O}, \mathrm{D}_{2} \mathrm{O}, \mathrm{C}_{2} \mathrm{H}_{2}, \mathrm{C}_{2} \mathrm{H}_{4}, \mathrm{C}_{4} \mathrm{H}_{6}, \mathrm{C}_{6} \mathrm{H}_{6}, \mathrm{CH}_{4}$ und $\mathrm{C}_{7} \mathrm{H}_{16}$ wurden relative Ionisationsquerschnitte gemessen. Die mit Quantenenergien unter 14,5 eV auftretenden, geladenen Bruchstücke dieser Moleküle wurden ebenfalls untersucht. Die Auflösung des Monochromators, $\Delta \lambda=1 \AA$, erlaubt es, Ionisierungspotentiale auf $\pm 0,005 \mathrm{eV}$ genau anzugeben.
\end{abstract}

Die Entwicklung intensiver Strahlungsquellen für das ferne Vakuum-Ultraviolett hat es in den letzten sechs Jahren möglich gemacht, Photoionisations- und Fragmentierungsprozesse von Molekülen auch in der Ionenkammer eines Massenspektrometers zu untersuchen. Aus den seit 1959 erschienenen Arbeiten ${ }^{1-7}$ läßt sich der rasche Fortschritt in dieser experimentellen Technik ablesen. Die Kombination eines Ultraviolett-Monochromators mit einem Massenspektrometer vereinigt die Vorteile der beiden älteren Untersuchungsmethoden von Molekülen, Massenspektrometrie und Ultraviolettspektroskopie.

Der allgemeine Aufbau der Apparatur ist in Abb. 1 dargestellt.

Licht von der Lichtquelle (L) tritt durch einen Strömungskanal und den Spalt (Se) in den Monochromator ein undt rifft dort auf dąs Konkavgitter (G). Hinter dem Austrittsspalt. (Sa) des Monochromators durchsetzt es den Ionisationsraum (IR) und wird dann mit Hilfe

* Jetzt Joint Institute for Laboratory Astrophysics, Boulder, Colorado 80304.

1 H. Hürzeler, M. G. Inghram u. J. D. Morrison, J. Chem. Phys. 28, 76 [1958].

2 B. Steiner, C. F. Giese u. M. G. Inghram, J. Chem. Phys. 34,189 [1961].

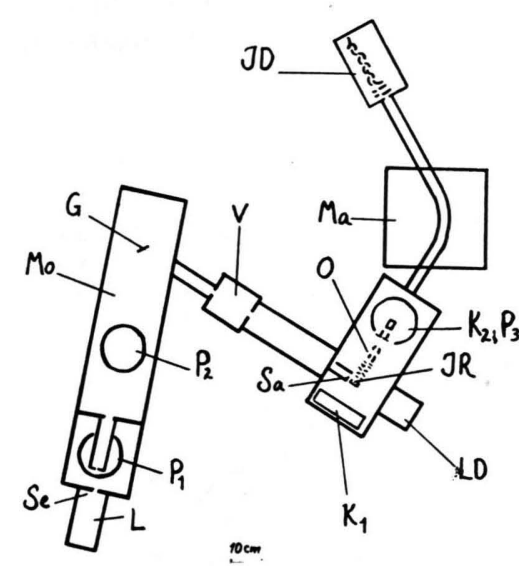

Abb. 1. Schematische Darstellung der Apparatur. L = Lichtquelle; Mo $=$ Monochromator; IR = Ionisationsraum; $\mathrm{LD}=$ Lichtdetektor; $\mathrm{Ma}=$ Magnet; ID = Ionendetektor; Se, Sa = Spalte des Monochromators; $\mathrm{G}=$ Gitter; $\mathrm{V}=$ Ventil, $\mathbf{O}=$ Ionenoptik; $\mathrm{P}_{1}, \mathrm{P}_{2}, \mathrm{P}_{3}=$ Diffusionspumpen; $\mathrm{K}_{1}, \mathrm{~K}_{2}=\mathrm{Kühl}$ fallen.

${ }^{3}$ F. A. Elder, C. F. Giese, B. Steiner u. M. G. Inghram, J. Chem. Phys. 36, 3292 [1962].

4 R. I. Schoen, J. Chem. Phys. 37, 2032 [1962].

5 E. Murad u. M.G. Inghram, J. Chem. Phys.40, 3263 [1964].

${ }^{6}$ F. J. Comes u. A. Elzer, Z. Naturforschg. 19 a, 721 [1964].

7 V. H. Dibeler, M. Krauss, R. M. Reese u. F. H. Harlee, J. Chem. Phys. 42, 3791 [1965]. 